\title{
Investigation of the femtosecond inverse Faraday effect using paramagnetic $\mathrm{Dy}_{3} \mathrm{Al}_{5} \mathrm{O}_{12}$
}

\author{
A. H. M. Reid, ${ }^{1, *}$ A. V. Kimel, ${ }^{1}$ A. Kirilyuk, ${ }^{1}$ J. F. Gregg, ${ }^{2}$ and Th. Rasing ${ }^{1}$ \\ ${ }^{1}$ Radboud University Nijmegen, Institute for Molecules and Materials, Heijendaalseweg 135, 6525 AJ Nijmegen, The Netherlands \\ ${ }^{2}$ Department of Condensed Matter Physics, Clarendon Laboratory, University of Oxford, Parks Road, Oxford OX1 3PU, United Kingdom
}

(Received 3 February 2010; published 8 March 2010)

\begin{abstract}
Pump-probe experiments on the rare-earth paramagnet $\mathrm{Dy}_{3} \mathrm{Al}_{5} \mathrm{O}_{12}$ demonstrate that coupling between light and magnetism on the subpicosecond time scale cannot be adequately described by the thermodynamic model of the inverse Faraday effect but instead must be described microscopically by stimulated magneto-Raman scattering. Light-induced paramagnetic resonance, predicted by the thermodynamic theory, is not observed in $\mathrm{Dy}_{3} \mathrm{Al}_{5} \mathrm{O}_{12}$, however, the formation of a coherent superposition between other magnetic sublevels of the $\mathrm{Dy}^{3+}$-ion's ground-state multiplet is measured. It is shown that coherence can only be induced between magnetic levels which are connected by the Raman selection rules.
\end{abstract}

DOI: $10.1103 /$ PhysRevB.81.104404

PACS number(s): 78.47.-p, 71.70.Ch, 78.20.Ls

\section{INTRODUCTION}

Femtosecond laser manipulation of spins is one of the most exciting new developments in condensed-matter physics, opening new possibilities for ultrafast recording and processing of magnetically stored information, ${ }^{1-5}$ and even offering a possible new avenue toward quantum computing. ${ }^{6}$ In several recent experiments, it has been demonstrated that an ultrashort laser pulse can act as an equally short magnetic field pulse, with a magnitude of several tesla; this has enabled spin resonances to be excited in ferrimagnetic garnets, ${ }^{7}$ antiferromagnets, ${ }^{4}$ and in an alloy of gadolinium-iron-cobalt. ${ }^{8}$ This optical manipulation of magnetism (optomagnetism) has sidestepped any previously held ultimate speed limit for the dynamics of magnetization. ${ }^{9}$ Heretofore, the understanding of these experiments has been based on the inverse Faraday effect (IFE). Phenomenologically, the IFE relates the optically induced field to the same magneto-optical susceptibility which determines the Faraday effect-rotation of polarization proportional to magnetization. The problem, however, is that such reciprocity between Faraday and inverse Faraday effects is strictly only defined in, or near, thermal equilibrium and therefore may not be valid for these highly nonequilibrium experiments performed on a subpicosecond time scale.

In this paper, we explore the nature of subpicosecond optomagnetism in a rare-earth paramagnet and demonstrate that coupling between light and spins at the ultrafast time scale cannot be wholly explained in terms of a thermodynamic inverse Faraday effect but instead must be microscopically described by stimulated Raman scattering from magnons. This interpretation is supported by the observation of specific Raman selection rules. In Sec. II of this paper, we discuss the IFE and the limitations of this theory with respect to the current experiments. Section III details an experimental investigation of the effect using a paramagnetic rare-earth aluminum garnet. The results of these experiments are discussed in Secs. IV and V. The limitations of comparing dynamic measurements to static properties are discussed in Sec. VI and in the final Sec. VII, conclusions are drawn.

\section{BACKGROUND TO THE INVERSE FARADAY EFFECT}

The possibility that magnetization could be generated by circularly polarized light was first proposed by Pitaevskii in a
1961 paper, ${ }^{10}$ however, the link to the Faraday effect was only later realized by Pershan. ${ }^{11}$ Using general arguments about the free energy in solids, Pershan predicted that the magnetization generated would be related to the same magneto-optical tensor that characterizes the long established Faraday effect. Further to this, Pershan and his coworkers went on to test these predictions and established experimentally that magnetization could indeed be generated by circularly polarized light. ${ }^{12}$ These experiments also verified that this induced magnetization correlated with the magneto-optical Verdet constant which characterizes the Faraday effect. In parallel with these experimental results, Pershan et al. went on to give a more in depth theory of the inverse Faraday effect and predicted several other related effects, such as magnetism generated by Raman scattering. ${ }^{13}$

The central premise of Pershan's theory of the inverse Faraday effect is that a free energy can be associated with a transparent medium subject to a time-varying electromagnetic field. The pertinent contributions to this free energy, for the IFE, takes the form of a product of three fields,

$$
\begin{aligned}
F= & 2 \operatorname{Re} \varepsilon_{0} \chi_{i j k}\left(\omega_{1}, \omega_{2}, \omega_{3}\right)\left[E_{i}^{*}\left(\omega_{1}\right) E_{j}\left(\omega_{2}\right) H_{k}\left(\omega_{3}\right)\right. \\
& \left.+E_{i}\left(\omega_{1}\right) E_{j}^{*}\left(\omega_{2}\right) H_{k}\left(\omega_{3}\right)\right],
\end{aligned}
$$

where $\chi_{i j k}$ is the magneto-optical susceptibility tensor and asterisks denote complex conjugates. Both the Faraday and inverse Faraday effects can be derived directly from this expression. The Faraday effect, a change in the electric polarization proportional to the magnetic-flux density, is obtained by using the definition for electric polarization, $P_{i}=$ $-\partial F / \partial E_{i}^{*}$. This gives the result, $P_{i}=\varepsilon_{0}\left(\chi_{i j k} E_{j} H_{k}+\chi_{j i k}^{*} E_{j} H_{k}^{*}\right)$. Analogously, the inverse Faraday effect comes about from the definition of a magnetization, $\mu_{0} M_{i}=-\partial F / \partial H_{i}^{*}$, which yields

$$
\mu_{0} M_{i}=-\varepsilon_{0} \chi_{i j k}^{*}\left(E_{j} E_{k}^{*}+E_{j}^{*} E_{k}\right),
$$

magnetization proportional to the optical fluence along the direction of propagation, the sign of which depends on the helicity of the optical field.

At this stage, it is worth reminding ourselves of the central issue: how does the inverse Faraday effect manifest on the subpicosecond time scale? In $\mathrm{DyFeO}_{3}$, Kimel et al. dem- 
onstrated the excitation of quasiferromagnetic and quasiantiferromagnetic resonances using pulses of circularly polarized light. ${ }^{4}$ The results were interpreted as the action of a $200 \mathrm{fs}$ light-induced magnetic field pulse, the sign of which reversed with a change from right-to-left-handed circular polarization - the key signature of the inverse Faraday effect. This reversal was observed as a $180^{\circ}$ phase shift of the induced resonances. In addition, Hansteen et al. observed similar results in ferrimagnetic lutetium iron garnet: the ability to optically induce ferrimagnetic resonance. ${ }^{14}$ In the all-optical switching experiments on alloys of $\mathrm{GdFeCo}$, opposite pump helicities are observed to write magnetic domains with different magnetization directions. ${ }^{5,15}$ Again, this is attributed to the action of an effective field either parallel or antiparallel to the optical beam.

It is apparent that there is a subtle, but fundamental, difference between the inverse Faraday effect invoked by Kimel et al. and Hansteen et al. to explain their ultrafast results, and that derived and measured by Pershan and his co-workers. The former always invokes an effective magnetic field, which acts on magnetism or spins while the latter is described in terms of an induced magnetization. Obviously such a distinction is not relevant in equilibrium, where, given enough time, magnetization will eventually align itself along the effective-field direction. However, this clearly cannot happen for femtosecond light pulses or even on longer time scales in materials with low magnetic damping. On very short time scales, the observable is not an induced magnetization but rather a light-induced effective field which would ultimately lead to magnetization. It is therefore tempting to simply divide the magnetization derived from Pershan's theory by the materials magnetic susceptibility to obtain the effective magnetic field. It is the rather simple conjecture that we test in this paper, we come to the conclusion that it is not generally valid.

\section{EXPERIMENTAL}

To test the premise that a subpicosecond pulse of circularly polarized light will generate an effective field proportional to the magneto-optical Verdet constant, a suitable magnetic system must be chosen on which to conduct the experiment. Our system of choice was paramagnetic dysprosium aluminium garnet $\left(\mathrm{Dy}_{3} \mathrm{Al}_{5} \mathrm{O}_{12}\right)$. Several reasons motivated this choice: first, unlike the rare-earth transition-metal compounds previously studied, the magnetic exchange coupling can be neglected in $\mathrm{Dy}_{3} \mathrm{Al}_{5} \mathrm{O}_{12}$, as can the effect of femtosecond pulses on this coupling; ${ }^{16}$ second, it is a transparent paramagnet and thus shows no discernible ultrafast demagnetization - $\mathrm{a}$ competing effect; ${ }^{1}$ third, $\mathrm{Dy}_{3} \mathrm{Al}_{5} \mathrm{O}_{12}$ possesses extremely strong spin-orbit coupling (a prerequisite for optomagnetic effects $\left.{ }^{17,18}\right)$; fourth, there are no complications due to the presence of photoinduced magnetic anisotropy as observed in iron garnets, ${ }^{14}$ fifth, at low temperatures, relaxation times are relatively long (several nanoseconds) and, therefore, no redistribution of population can be expected within the pulse duration; ${ }^{19}$ finally, sixth, $\mathrm{Dy}_{3} \mathrm{Al}_{5} \mathrm{O}_{12}$ has extremely strong magneto-optical properties - a measurement of $\mathrm{Dy}_{3} \mathrm{Al}_{5} \mathrm{O}_{12}$ 's Verdet constant

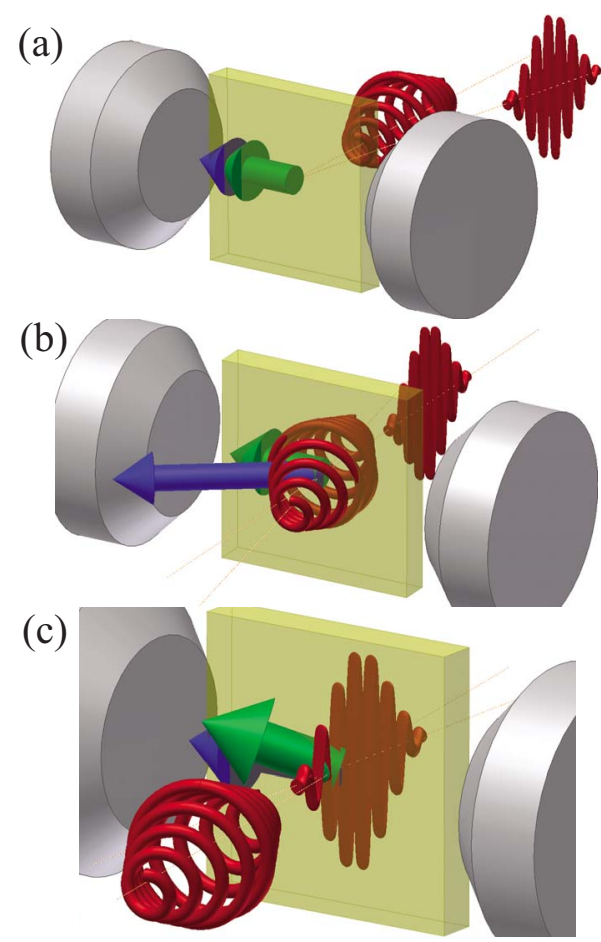

FIG. 1. (Color online) The pump-probe setup. (a) An external field (blue/dark gray arrow) was applied to the $\mathrm{Dy}_{3} \mathrm{Al}_{5} \mathrm{O}_{12}$ sample to induce magnetization [green (light gray) arrow] in the sample plane. (b) The circularly polarized pump pulse deflects the effective field out of the sample plain via the inverse Faraday effect. The magnetization should precess around this modified field during the pulse. (c) The magnetization, no longer aligned along the original external field, precesses around it with an angle proportional to the strength of the inverse Faraday effect. This precession is measured by observing the Faraday rotation of a linearly polarized probe pulse.

at the experimental wavelength and temperature gave a value of $1.14 \times 10^{3} \mathrm{rad} \mathrm{T}^{-1} \mathrm{~m}^{-1}$. In comparison, the fully saturated Faraday rotation in the iron-garnet films measured by Hansteen et al. was approximately $650 \mathrm{rad} \mathrm{m}^{-1}$.7,14

To investigate the response of $\mathrm{Dy}_{3} \mathrm{Al}_{5} \mathrm{O}_{12}$ to circularly polarized light, a 1-mm-thick [001] crystalline plate was mounted in a continuous-He-flow cold-finger cryostat, with optical access, and cooled to $10 \mathrm{~K}$. To observe the action of the effective magnetic field, we duplicated the experiment of Hansteen et al. ${ }^{14}$ Figure 1 shows the experimental geometry. The $\mathrm{Dy}_{3} \mathrm{Al}_{5} \mathrm{O}_{12}$ crystal is arranged between the pole pieces of an electromagnet, which induced magnetization in the sample plane. The pump beam consists of a $50 \mathrm{fs}$ pulse of circularly polarized light with a wavelength centered at 790 $\mathrm{nm}$. The pulse was focused down to a spot size of approximately $100 \mu \mathrm{m}$ diameter and arranged to pass through the sample at about $5^{\circ}$ from normal incidence, see Fig. 1(a).

\section{RESULTS}

According to the supposition, the pump creates a strong effective magnetic field which tips the overall field ( $\mathbf{B}_{\text {pump }}$ $+\mathbf{B}_{\text {ext }}$ ) away from the magnetization direction, see Fig. 1(b). 
During the pulse, $\mathrm{Dy}_{3} \mathrm{Al}_{5} \mathrm{O}_{12}$ 's magnetization will precess in this modified effective field, moving away from the appliedfield direction. When the pump pulse has exited the $\mathrm{Dy}_{3} \mathrm{Al}_{5} \mathrm{O}_{12}$ crystal, the magnetization should no longer be aligned along the external magnetic field, see Fig. 1(c), and thus it will precess around this field. The precessing magnetization can be measured using the time-delayed probe pulse and should be observable until relaxation takes place in about 3-10 ns. ${ }^{19}$

Before conducting the experiment, it is worth exploring what the theory predicts. Equation (2) can be rewritten in terms of the Verdet constant, ${ }^{13}$

$$
\mathbf{M}=\frac{V \lambda_{0}}{2 \pi c}\left(I_{\mathrm{R}}-I_{\mathrm{L}}\right) \hat{\mathbf{k}}
$$

where $\mathbf{M}$ is the induced magnetization $\left(\mathrm{A} \mathrm{m}^{-1}\right), V$ is the Verdet constant $\left(\operatorname{rad~T}^{-1} \mathrm{~m}^{-1}\right) ; \lambda_{0}$ is the optical wavelength; $c$ is the speed of light; $I_{\mathrm{R}}$ and $I_{\mathrm{L}}$ are the optical intensities $\left(\mathrm{J} \mathrm{m}^{-2} \mathrm{~s}^{-1}\right)$, and $\hat{\mathbf{k}}$ is a unit vector in the direction of propagation. Note that the form of Eq. (3) is the same in S.I. and c.g.s. units.

Using Eq. (3), the magnetization induced in $\mathrm{Dy}_{3} \mathrm{Al}_{5} \mathrm{O}_{12}$, for continuous excitation, can be estimated to be 1.9 $\times 10^{3} \mathrm{~A} \mathrm{~m}^{-1}$ (the laser fluence used was $200 \mathrm{~mJ} \mathrm{~cm}^{-2}$, which was delivered in $50 \mathrm{fs}$ ). The volume magnetic susceptibility $(\chi)$ of $\mathrm{Dy}_{3} \mathrm{Al}_{5} \mathrm{O}_{12}$ at $10 \mathrm{~K}$ is $8.4 \times 10^{-2}$ (Ref. 20). Thus the effective magnetic field acting on the garnet is calculated from the relation $\mathbf{B}=\mu_{0} \mathbf{M} / \chi$; this gives a value of $\mathbf{B}_{\text {pump }}=0.28 \mathrm{~T}$, the same order of magnitude as that observed in the iron garnet measured by Hansteen et al. ${ }^{14}$ Using the duration of this field pulse (approximately $50 \mathrm{fs}$ ), $\mathrm{Dy}_{3} \mathrm{Al}_{5} \mathrm{O}_{12}$ 's gyromagnetic ratio $(g=18.2$, Ref. 20$)$ and the externally applied magnetic field $\left(\mathbf{B}_{\text {ext }}=0.3 \mathrm{~T}\right)$ the deflection of the magnetization was calculated to be at an angle of $1.3^{\circ}$ from the external field. Such a deviation would cause a periodic oscillation in the probe Faraday rotation with an amplitude of $0.4^{\circ}$ and frequency of $76.5 \mathrm{GHz}$, easily resolvable in the experimental setup.

The results of the experiment were, however, quite different. The application of a circularly polarized pump pulse caused a large deviation in the Faraday rotation of the probe (see Fig. 2) that decayed within 200 fs. This effect is observed in most materials and is due to the induction of a transient polarization. Following this transient, a strong oscillation in the sample's Faraday rotation becomes apparent, as predicted. However, the frequency of this oscillation, measured to be $2.17 \mathrm{THz}$, is nearly 30 times higher than expected for magnetization precessing in the external field. Further experiments showed no measurable variation in this frequency for different orientations or values of the external field. A change from right-to-left-handed circular polarization, however, did cause the $180^{\circ}$ of phase shift associated with the inverse Faraday effect (see Fig. 2). The oscillation also disappeared for a linearly polarized pump pulse and did not appear in the sample's transmissivity [see Fig. 5(b)] good evidence that the effect was magneto-optic in origin. The inset of Fig. 2 shows the results for longer time scales, up to $1.2 \mathrm{~ns}$. In these measurements, only a linear change

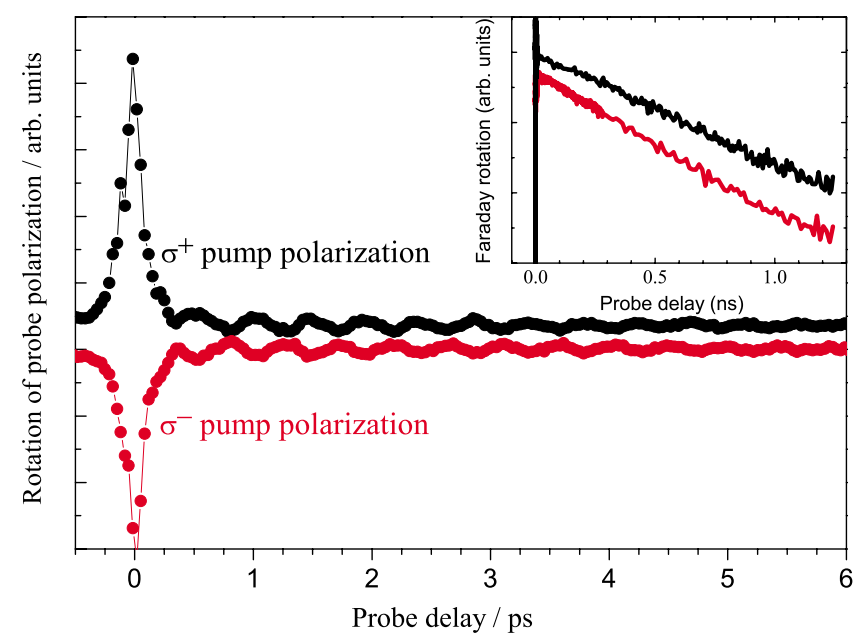

FIG. 2. (Color online) The time-resolved Faraday rotation of $\mathrm{Dy}_{3} \mathrm{Al}_{5} \mathrm{O}_{12}$ after excitation with right- and left-circularly polarized light $\left(\sigma^{-}\right.$and $\sigma^{+}$, respectively). The inset shows the response over the first nanosecond.

with time of the Faraday signal is observed, which was associated with heating of the crystal by the laser pulse.

To explore further whether these results were related to the thermodynamic description of the IFE, we compared the initial amplitudes of the observed oscillation with static measurements of the material's Verdet constant over a range of temperatures. This data, plotted in Fig. 3, shows that the two have very different temperature dependencies. The Verdet constant follows a classic Curie-Weiss law behavior since the Faraday effect scales with the paramagnetic susceptibility. However, the ultrafast process does not display this behavior; it instead decreases steadily and disappears completely at $100 \mathrm{~K}$. It should be noted that the effective field generated by the IFE is not predicted to vary with temperature. This is because the Verdet constant and the magnetic susceptibility

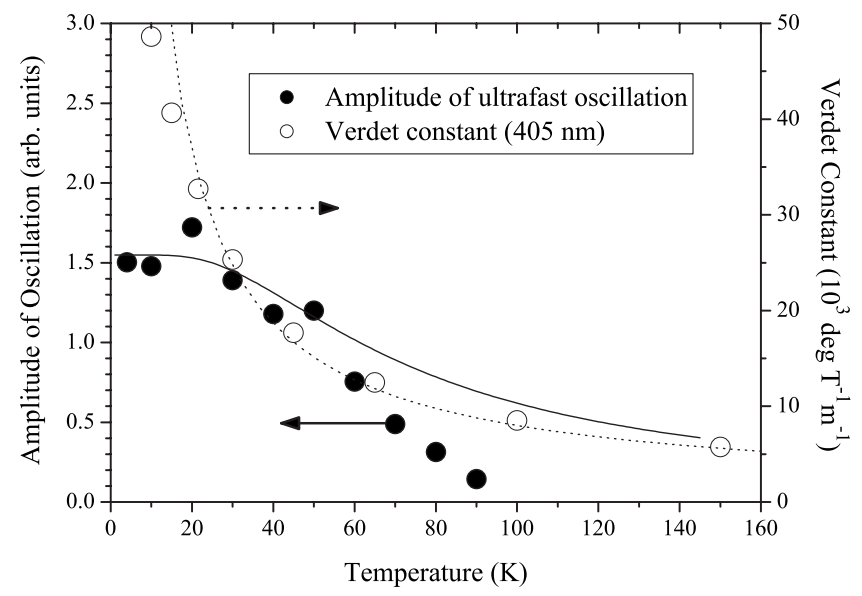

FIG. 3. Static measurements of the Verdet constant on $\mathrm{Dy}_{3} \mathrm{Al}_{5} \mathrm{O}_{12}$ are compared to the amplitude of the high-frequency oscillation. The measured temperature dependence of the Verdet constant fits a Curie-law behavior (dashed line), this does not hold for the amplitude of the high-frequency oscillation. It instead shows agreement below $50 \mathrm{~K}$ with the predicted Raman efficiency due to thermal populations in the excited states (solid line). 
both have the same dependence on temperature; their ratio, upon which the effective field depends, thus remains approximately constant.

\section{DISCUSSION}

Clearly it is impossible to explain the experimental results by invoking the present description of the IFE. This presents two mysteries: what alternative physical mechanism can explain the observed results and why does the effective-field argument break down? These questions will be tackled in turn. First, how to explain the origin of the observed highfrequency oscillation? As its frequency does not fit with any Zeeman splitting of the dysprosium ion in a magnetic field, other interactions within the garnet need to be considered. The ionic ground state of the dysprosium ion in $\mathrm{Dy}_{3} \mathrm{Al}_{5} \mathrm{O}_{12}$ is a $J=15 / 2$ multiplet ( $L=5$ and $S=5 / 2$ coupled by the spinorbit interaction). This multiplet has a degeneracy of $2 J+1$ (16) which is partially lifted by the crystal field. ${ }^{21}$ In $\mathrm{Dy}_{3} \mathrm{Al}_{5} \mathrm{O}_{12}$, the crystal field splits this multiplet into eight Kramers' doublets. A comparison with previously published spectral measurements of $\mathrm{Dy}_{3} \mathrm{Al}_{5} \mathrm{O}_{12}$ revealed that the frequency exactly matches the first of these crystal-field-split doublet states above the ground state. ${ }^{22}$ This strongly points to the conclusion that the pump pulse causes the formation of a coherent superposition of ground and excited states, which leads to beating at a frequency corresponding to their energy difference.

The creation of coherence requires an interaction that connects the atomic levels involved. Direct mixing by electric dipole transitions between the crystal-field-split states within the bottom $J$ multiplet are strictly parity forbidden so indirect transitions-occurring through the mixing of nonresonant excited states - are the only available mechanism; these are often referred to as electronic Raman transitions. To understand the processes involved, it is useful to consider the simple three-level system shown in Fig. 4. In general, the scattering rate can be written down from perturbation theory,

$$
S \propto \frac{1}{\hbar^{2}}\left|\sum_{e}\left(\frac{\langle f|\hat{V}| e\rangle\langle e|\hat{V}| g\rangle}{\omega_{e g}-\omega}+\frac{\langle f|\hat{V}| e\rangle\langle e|\hat{V}| g\rangle}{\omega_{e g}+\omega}\right)\right|^{2},
$$

where, $\hat{V}$ is the electric dipole operator, $g, e$, and $f$ denote the ground, excited, and final states of the system, and $\omega_{e g}$ and $\omega$ correspond to the excited state and optical frequencies, respectively.

Further evidence that we are observing a Raman transition between two levels can be gleaned from what occurs beyond the coherence time. The quantum beating in the Faraday effect decays with a time constant $\tau=3.5 \mathrm{ps,} \mathrm{most} \mathrm{likely}$ caused by dipolar coupling to the paramagnetic spin bath. The signal does not return to the prepump value but instead a change in the Faraday effect persists with opposite sign for each circular polarization of the pump. It is this that contributes to the offset between the curves seen in the inset of Fig. 2 , however, it is most clearly observed for time scales where the coherence has vanished but still shorter than the thermal relaxation times [see Fig. 5(a)]. This change did not appear in the transmissivity data [Fig. 5(b)], again suggesting

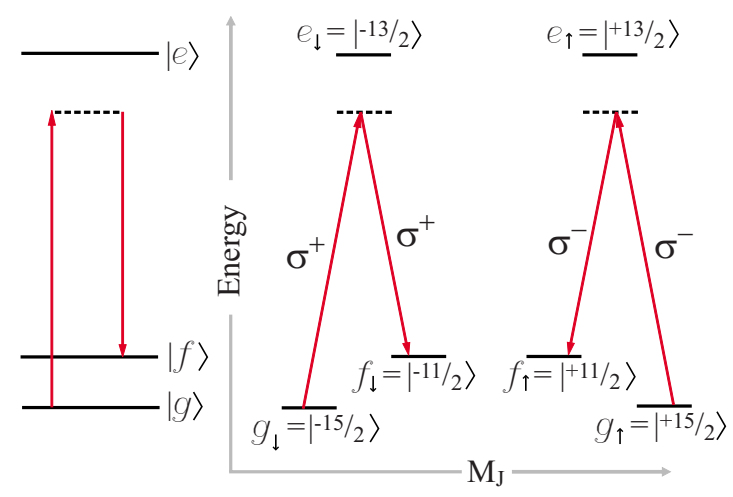

FIG. 4. (Color online) A schematic of the Raman process on an energy-level diagram. The absorption of a photon causes the system to undergo a virtual transition to the state $|e\rangle$. This is followed by a stimulated emission that coherently puts the system into its final state $|f\rangle$. On the right, we show a simplified explanation of the process in terms of the $M_{\mathrm{J}}$ selection rules. Circular polarizations act as raising and lowering operators on the magnetic quantum number; $\sigma^{+}$light operates on the $|g \downarrow\rangle$ connecting it to the $|f \downarrow\rangle$, which contains the correct $M_{\mathrm{J}}$ components. The same process happens for the $|g \uparrow\rangle$ with $\sigma^{-}$polarized light.

magneto-optic origins. It is noted that a similar result was observed in the measurements of Kimel et al., however, its origin has remained an open question. ${ }^{4}$ We associate this

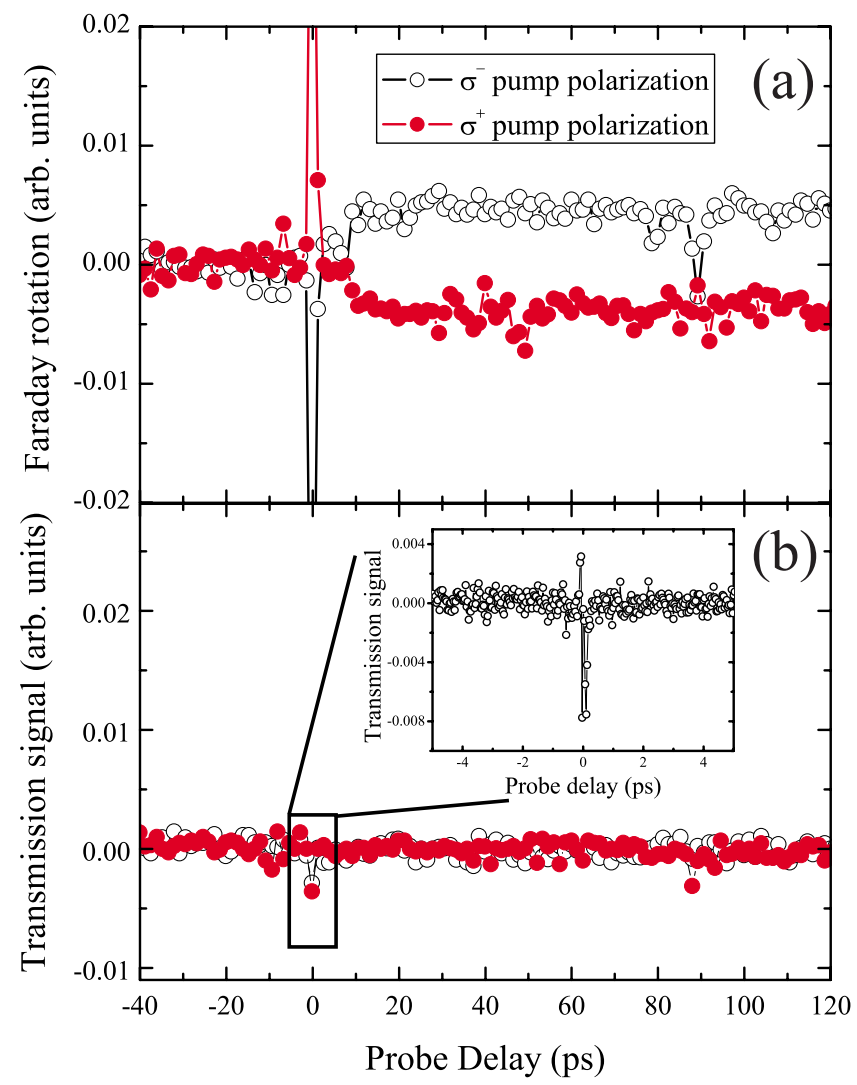

FIG. 5. (Color online) (a) The longer-lasting steplike change in the Faraday effect after circularly polarized pump pulses and (b) the total intensity measured at the diode detector (transmissivity) as a function of the pump-probe delay. 
effect with the creation of a diagonal element in the density matrix-population transfer to the excited state. At $10 \mathrm{~K}$, no discernible decay can be resolved in this steplike change in the Faraday signal within the maximum delay time permitted by the experiment $(1.4 \mathrm{~ns})$. This matches the expectation of a relatively long $T_{1}$ time at low temperatures. ${ }^{23}$ Such a transfer of population between levels is expected to cause a small change in the magnetization of the garnet. For example, in the case of the $\sigma^{+}$scattering, the net moment contributed by the ground state is increased by scattering from the $\left|g_{\downarrow}\right\rangle$ ground state, into the $\left|f_{\downarrow}\right\rangle$ excited state, resulting in a net increase in $M_{\mathrm{J}}$.

It is well known that impulsively stimulated Raman scattering can take place, if the duration of the pulse is short compared to the period of the generated excitation. This is the case for ground $(g)$ and first excited states $(f)$ in $\mathrm{Dy}_{3} \mathrm{Al}_{5} \mathrm{O}_{12}$, and is the most likely mechanism by which the coherence is created. However, it is now prudent to return to the second question: why is no coherence induced between the ground-state levels $\left(g_{\uparrow}\right.$ and $\left.g_{\downarrow}\right)$ ? These levels also fulfill the requirements that their energy splitting is within the bandwidth of the pulse and are in no way fundamentally different to the excited states. The question becomes, why does the excited-state resonance appear and not the groundstate resonance. The explanation is found by considering the angular momentum selection rules governing the Raman process. These may be determined by applying the WignerEckart theorem to the matrix elements in Eq. (4); each matrix element corresponds to an electric dipole transition, with selection rules, $\Delta J= \pm 1$ and $\Delta M_{\mathrm{J}}= \pm 1$. The overall selection rule for $g \rightarrow e \rightarrow f$ scattering must be $\Delta J=0$ and $-2 \leq \Delta M_{\mathrm{J}}$ $\leq 2$.

The transitions induced by circularly polarized light act as a ladder operator for the magnetic quantum number $M_{\mathrm{J}}$. A right-hand circularly polarized pump $\left(\sigma^{-}\right)$will act to lower $M_{\mathrm{J}}$ while left-hand polarized light $\left(\sigma^{+}\right)$will act to raise $M_{\mathrm{J}}$. Applying the selection rules to the ground state of $\mathrm{Dy}_{3} \mathrm{Al}_{5} \mathrm{O}_{12}$ shows that scattering is not possible between $\left|g_{\uparrow}\right\rangle$ and $\left|g_{\downarrow}\right\rangle$ states, as shown in Fig. 4. In reality, however, the crystal field acts to smear the magnetic quantum-number components in the ground state. Considering a dysprosium moment with local magnetic axis along the applied-field direction, a diagonalization of the crystal-field Hamiltonian, given by Wadsack et al., gives the spin-up ground state to be $\left|g_{\uparrow}\right\rangle=70 \%\left|\frac{15}{2}\right\rangle+26 \%\left|\frac{11}{2}\right\rangle+1 \%\left|\frac{7}{2}\right\rangle+3 \%\left|\frac{3}{2}\right\rangle \cdots$ while the spindown ground state is $\left|g_{\downarrow}\right\rangle=70 \%\left|\frac{-15}{2}\right\rangle+26 \%\left|\frac{-11}{2}\right\rangle+1 \%\left|\frac{-7}{2}\right\rangle$ $+3 \%\left|\frac{-3}{2}\right\rangle+\cdots .22$ The transfer between $\left|g_{\uparrow}\right\rangle$ and $\left|g_{\downarrow}\right\rangle$ is therefore not forbidden. However, calculation shows that the scattering rate for the $\left|g_{\uparrow}\right\rangle \rightarrow\left|f_{\uparrow}\right\rangle$ transfer is more than two orders of magnitude larger than the $\left|g_{\uparrow}\right\rangle \rightarrow\left|g_{\downarrow}\right\rangle$ transitions. We thus surmise from our results that an allowed Raman transition is required between magnetic levels to observe ultrafast optomagnetism.

In light of understanding the origin of the oscillation, we reexamined its dependence on temperature. The decay time constant and oscillation frequency are shown in Fig. 6 as a function of temperature. The decay rate does not show a strong dependence on temperature. This indicates that the decoherence time is not dominated by phonon interactions but more likely depends on magnetic interactions and crystal defects.

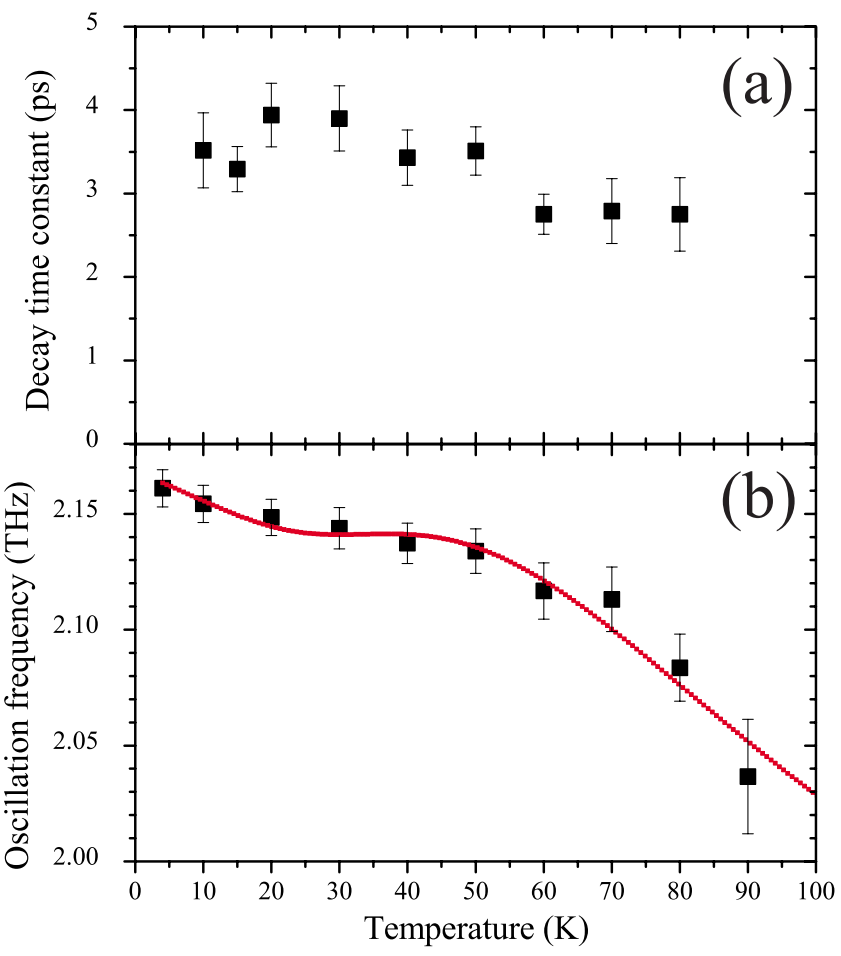

FIG. 6. (Color online) (a) The time taken for the $2.1 \mathrm{THz}$ oscillation to decay time and (b) the frequency of oscillation as a function of temperature (line shows fit model).

To explain the change in oscillation frequency with temperature, it is necessary to examine the electronic structure. As the temperature is increased, the populations of the lowest-lying excited states, which occur at energies corresponding to 104,164 , and $268 \mathrm{~K}$, become important. Population in these states can cause a modification of the crystal field due to the transfer of population to different ligands. These modify various contributions to the crystal field such as wave-function overlap, covalence, and nascent covalent bonds. ${ }^{24} \mathrm{~A}$ simple parametrization in terms of these level occupations can easily account for the variation in frequency as is shown in Fig. 6(b).

\section{LIMITATIONS OF THE EFFECTIVE FIELD PICTURE}

Now that the origin of the induced coherence has been established, it is worth returning to the problem of why there are limits on determining the inverse Faraday effect from static measurements. First, it should be pointed out that the inverse Faraday effect should indeed induce magnetization in $\mathrm{Dy}_{3} \mathrm{Al}_{5} \mathrm{O}_{12}$ if given sufficient time. Rare-earth paramagnetic glasses have been tested ${ }^{12}$ and showed agreement with the predictions of Eq. (3). In addition, the response of terbium gallium garnet $\left(\mathrm{Tb}_{3} \mathrm{Ga}_{5} \mathrm{O}_{12}\right)$, a very similar material to $\mathrm{Dy}_{3} \mathrm{Al}_{5} \mathrm{O}_{12}$, has been measured and was found to display an optically induced magnetization consistent with Eq. (3) (Ref. 25).

This apparent contradiction can be resolved by considering the microscopic origins of the effect. In the garnet structure, there are six distinct rare-earth $(c)$ sites; these have identical local symmetries but each has a different orienta- 


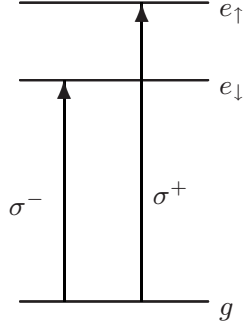

(a)

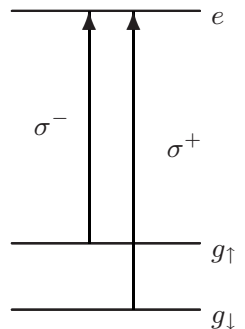

(b)
FIG. 7. Illustration of the diamagnetic-type contribution to (a) the Faraday effect and (b) the paramagnetic-type contribution.

tion with respect to the crystallographic axes. ${ }^{26}$ These six sites can be subdivided into three pairs, each of which have their primary magnetic axes orientated along one of the crystallographic directions. Thus while the $\mathrm{Dy}_{3} \mathrm{Al}_{5} \mathrm{O}_{12}$ crystal displays isotropic magnetic properties, individual dysprosium moments are actually highly anisotropic. ${ }^{27}$ Here we emphasis that the Faraday and inverse Faraday effects are local in nature, and thus the action must be considered on individual magnetic moments. Each pair of sites will "see" a different effective field depending on their orientation. Those sites which experience the strong inverse Faraday field, predicted by statics, have a negligible magnetic susceptibility perpendicular to this directions, along the external field; thus no induced moment which can precess around the IFE field. On the other hand, those sites with an induced magnetic moment do not experience any a strong IFE because, as shown in the previous section, there is no Raman connection between their $\left|g_{\uparrow}\right\rangle$ and $\left|g_{\downarrow}\right\rangle$ ground states.

While the laser-induced field acts strongly only with a fraction of the magnetic moments, these are also the moments which have a high susceptibility along the field direction, and thus are responsible for the induced magnetization. The situation is different in quasistatic measurements, where there is no need to consider the local anisotropy of the magnetic moments.

It should be noted that there may be other limits to determining the effective field created by the inverse Faraday effect from static measurements of the Faraday effect. This is due to the fact that there is not a single microscopic origin of the Faraday effect. These origins are not trivial and have been discussed at length by many authors. ${ }^{28-31}$ However, general observations can be made: usually two contributions to the Faraday effect are distinguished, namely, the paramagnetic contribution (which results from the influence of magnetism on ground states) and the diamagnetic contribution (which is the result of the magnetic influence on excited states). Figure 7 illustrates the difference between these. It is normal for both of these contributions to be present but usually one dominates the response.

In the discussions of the inverse Faraday effect, only paramagnetic contributions have been explicitly considered (interaction between ground states); this is a presumption of Pershan's microscopic theory of the inverse Faraday effect, ${ }^{13}$ though not of Eq. (2). A diamagnetic contribution to the inverse Faraday effect should also occur. However, it is unclear if this is physically meaningful as there is no lasting influence on the ground state for nonresonant excitation. In fact, this diamagnetic inverse Faraday effect is essentially what was originally derived by Pitaevskij, as the system he considered had no ground-state magnetization. The experimental measurements on $\mathrm{Dy}_{3} \mathrm{Al}_{5} \mathrm{O}_{12}$ may also show this diamagnetic inverse Faraday effect; the transient observed at zero time delay in Fig. 2 should contain this effect, however, separating the true magnetic part from changes in the magnetic-optical susceptibility during strong laser excitation is a formidable problem. It therefore may be necessary to remove the diamagnetic contribution from static magnetooptical constants which are to be used as a basis to predict the inverse Faraday response. Certainly, care must be taken with respect to the microscopic origins as not all may contribute to the effective field.

\section{CONCLUSIONS}

In this paper, we have attempted to resolve whether or not the thermodynamical theory of the inverse Faraday effect is still valid on a subpicosecond time scale. An experimental and theoretical examination was undertaken which showed that, in general, a simple correspondence cannot be expected. We have demonstrated that in $\mathrm{Dy}_{3} \mathrm{Al}_{5} \mathrm{O}_{12}$, the effective field predicted by the magneto-optical Verdet constant cannot be observed for excitation by a 50 fs pulse. However, the observed formation of a terahertz-frequency quantum coherence between the material's magnetic sublevels indicates the presence of stimulated Raman scattering. Further examination of the selection rules governing the Raman process showed that direct scattering between the ground-state doublet is expected to be much weaker than to other levels. It is argued that a viable Raman connection between magnetic levels is a necessary requirement for optomagnetism. Furthermore, in choosing materials to exploit optomagnetism a greater importance must be placed on the Raman selection rules which link magnetic levels rather than the strength of static magneto-optical effects which are not necessarily a reliable indicator.

\section{ACKNOWLEDGMENTS}

The authors wish to thank C. D. Stanciu, F. Hansteen, A. M. Kalashnikova, A. T. Boothroyd, G. A. Brooker, R. L. Cone, and W. P. Wolf for useful discussions. This work was supported by the European networks UltraMagnetron (NMP3-SL-2008-214469), FANTOMAS (214810), and DYNAMICS, and the Dutch national organizations for scientific research FOM and NWO. 
*a.reid@science.ru.nl

${ }^{1}$ E. Beaurepaire, J.-C. Merle, A. Daunois, and J.-Y. Bigot, Phys. Rev. Lett. 76, 4250 (1996).

${ }^{2}$ B. Koopmans, M. van Kampen, and W. J. M. de Jonge, J. Phys.: Condens. Matter 15, S723 (2003).

${ }^{3}$ G. Ju, J. Hohlfeld, B. Bergman, R. J. M. van de Veerdonk, O. N. Mryasov, J.-Y. Kim, X. Wu, D. Weller, and B. Koopmans, Phys. Rev. Lett. 93, 197403 (2004).

${ }^{4}$ A. V. Kimel, A. Kirilyuk, P. A. Usachev, R. V. Pisarev, A. M. Balbashov, and T. Rasing, Nature (London) 435, 655 (2005).

${ }^{5}$ C. D. Stanciu, F. Hansteen, A. V. Kimel, A. Kirilyuk, A. Tsukamoto, A. Itoh, and T. Rasing, Phys. Rev. Lett. 99, 047601 (2007).

${ }^{6}$ S. A. Wolf, D. D. Awschalom, R. A. Buhrman, J. M. Daughton, S. von Molnar, M. L. Roukes, A. Y. Chtchelkanova, and D. M. Treger, Science 294, 1488 (2001).

${ }^{7}$ F. Hansteen, A. Kimel, A. Kirilyuk, and T. Rasing, Phys. Rev. Lett. 95, 047402 (2005).

${ }^{8}$ C. D. Stanciu, F. Hansteen, A. V. Kimel, A. Tsukamoto, A. Itoh, A. Kirilyuk, and T. Rasing, Phys. Rev. Lett. 98, 207401 (2007).

${ }^{9}$ I. Tudosa, C. Stamm, A. B. Kashuba, F. King, H. C. Siegmann, J. Stöhr, G. Lu, and D. Weller, Nature (London) 428, 831 (2004).

${ }^{10}$ L. P. Pitaevskiı̆, Sov. Phys. JETP 12, 1008 (1961).

${ }^{11}$ P. S. Pershan, Phys. Rev. 130, 919 (1963).

${ }^{12}$ J. P. van der Ziel, P. S. Pershan, and L. D. Malmstrom, Phys. Rev. Lett. 15, 190 (1965).

${ }^{13}$ P. S. Pershan, J. P. van der Ziel, and L. D. Malmstrom, Phys. Rev. 143, 574 (1966).

${ }^{14}$ F. Hansteen, A. Kimel, A. Kirilyuk, and T. Rasing, Phys. Rev. B 73, 014421 (2006).
${ }^{15}$ K. Vahaplar, A. M. Kalashnikova, A. V. Kimel, D. Hinzke, U. Nowak, R. Chantrell, A. Tsukamoto, A. Itoh, A. Kirilyuk, and T. Rasing, Phys. Rev. Lett. 103, 117201 (2009).

${ }^{16}$ F. Dalla Longa, J. T. Kohlhepp, W. J. M. de Jonge, and B. Koopmans, J. Appl. Phys. 99, 08F304 (2006).

${ }^{17}$ A. Rebei and J. Hohlfeld, Phys. Lett. A 372, 1915 (2008).

${ }^{18}$ R. Gómez-Abal, O. Ney, K. Satitkovitchai, and W. Hübner, Phys. Rev. Lett. 92, 227402 (2004).

${ }^{19}$ I. Nowik and H. H. Wickman, Phys. Rev. 140, A869 (1965).

${ }^{20}$ M. Ball, W. P. Wolf, and A. F. G. Wyatt, J. Appl. Phys. 35, 937 (1964).

${ }^{21}$ B. G. Wybourne, Spectroscopic Properties of Rare Earths (Interscience, New York, 1965).

${ }^{22}$ R. L. Wadsack, J. L. Lewis, B. E. Argyle, and R. K. Chang, Phys. Rev. B 3, 4342 (1971).

${ }^{23}$ C.-Y. Huang, Phys. Rev. 139, A241 (1965).

${ }^{24}$ S. Hüfner, Optical Spectra of Transparent Rare Earth Compounds (Academic, New York, 1978).

${ }^{25}$ M. Y. A. Raja, D. Allen, and W. Sisk, Appl. Phys. Lett. 67, 2123 (1995).

${ }^{26}$ D. P. Landau and B. E. Keen, Phys. Rev. B 19, 4805 (1979).

${ }^{27}$ P. Grünberg, S. Hüfner, E. Orlich, and J. Schmitt, Phys. Rev. 184, 285 (1969).

${ }^{28}$ P. Pershan, J. Appl. Phys. 38, 1482 (1967).

${ }^{29}$ W. A. Crossley, R. W. Cooper, J. P. Page, and R. P. van Stapele, Phys. Rev. 181, 896 (1969).

${ }^{30}$ P. N. Argyres, Phys. Rev. 97, 334 (1955).

${ }^{31}$ A. K. Zvezdin and V. A. Kotov, Modern Magnetooptics and Magnetooptical Materials, Studies in Condensed Matter Physics (Insitute of Physics, Berkshire, 1997). 